\title{
Advantages of diabetic tractional retinal detachment repair
}

\author{
This article was published in the following Dove Press journal: \\ Clinical Ophthalmology \\ 23 October 2015 \\ Number of times this article has been viewed
}

\section{Amir Sternfeld \\ Ruth Axer-Siegel \\ Hadas Stiebel-Kalish \\ Dov Weinberger \\ Rita Ehrlich}

Department of Ophthalmology, Rabin Medical Center, Beilinson Campus, Petach Tikva, Israel
Correspondence: Amir Sternfeld Department of Ophthalmology, Rabin Medical Center, 39 Jabotinski Street, Petah Tikva 49100, Israel

Tel +9723937 6101

Fax+97239219084

Email shtery@gmail.com
Purpose: To evaluate the outcomes and complications of patients with diabetic tractional retinal detachment (TRD) treated with pars plana vitrectomy (PPV).

Patients and methods: We retrospectively studied a case series of 24 eyes of 21 patients at a single tertiary, university-affiliated medical center. A review was carried out on patients who underwent PPV for the management of TRD due to proliferative diabetic retinopathy from October 2011 to November 2013. Preoperative and final visual outcomes, intraoperative and postoperative complications, and medical background were evaluated.

Results: A $23 \mathrm{G}$ instrumentation was used in 23 eyes (95.8\%), and a $25 \mathrm{G}$ instrumentation in one (4.2\%). Mean postoperative follow-up time was 13.3 months (4-30 months). Visual acuity significantly improved from logarithm of the minimum angle of resolution (LogMAR) 1.48 to LogMAR $1.05(P<0.05)$. Visual acuity improved by $\geq 3$ lines in $75 \%$ of patients. Intraoperative complications included iatrogenic retinal breaks in seven eyes $(22.9 \%)$ and vitreal hemorrhage in nine eyes (37.5\%). In two eyes, one sclerotomy was enlarged to $20 \mathrm{G}(8.3 \%)$. Postoperative complications included reoperation in five eyes $(20.8 \%)$ due to persistent subretinal fluid $(n=3)$, vitreous hemorrhage $(n=1)$, and dislocated intraocular lens $(n=1)$. Thirteen patients $(54.2 \%)$ had postoperative vitreous hemorrhage that cleared spontaneously, five patients $(20.8 \%)$ required antiglaucoma medications for increased intraocular pressure, seven patients $(29.2 \%)$ developed an epiretinal membrane, and two patients (8.3\%) developed a macular hole.

Conclusion: Patients with diabetic TRD can benefit from PPV surgery. Intraoperative and postoperative complications can be attributed to the complexity of this disease.

Keywords: diabetic retinopathy, proliferative vitreoretinopathy, retinal detachment, vitrectomy, vitreoretinal surgery

\section{Introduction}

End-stage diabetic eye disease is an important cause of severe visual impairment in the working-age group. ${ }^{1}$ One of the feared ocular complications of diabetes mellitus is proliferative diabetic retinopathy (PDR) with tractional retinal detachment (TRD). ${ }^{2,3}$ When involving or threatening the macula or when accompanied by persistent vitreous hemorrhage $(\mathrm{VH}), \mathrm{TRD}$ is an indication for surgical intervention by pars plana vitrectomy (PPV). ${ }^{4}$

The modern era of vitrectomies began with the introduction of $20 \mathrm{G}$ instrumentation in 1975 by O'Malley and Heintz. ${ }^{5}$ It was a few decades later, a paradigm shift toward small-gauge vitrectomy (SGV) began in 2002 and 2004, with the first- and second-generations of $25 \mathrm{G}$ instrumentations developed by Fujii et al ${ }^{6}$ and Lesnoni et al, ${ }^{7}$ respectively. In 2005, Eckardt ${ }^{8}$ developed the $23 \mathrm{G}$ instrumentation.

First-generation $25 \mathrm{G}$ was used only for surgeries that did not require extensive dissection, such as epiretinal membrane peeling, macular pathologies, simple VH, or vitreomacular tractions. Its limited usage was due to several factors including excessive 
flexibility of the instruments that made some surgical maneuvers more difficult, low flow rates, less efficient cutters, and wound leakage-associated complications (postoperative hypotony and endophthalmitis) that were related to the sutureless closure of the ports. ${ }^{9,10}$

Second-generation $25 \mathrm{G}$ and $23 \mathrm{G}$ instruments were designed to overcome these limitations through increased rigidity, better fluidics, and a larger variety of instruments. This allowed the management of more complicated surgeries such as TRD, dense $\mathrm{VH}$, and rhegmatogenous retinal detachment. ${ }^{10-12}$

Diabetic vitrectomy holds significant risk for surgically induced complications, among which are intraoperative bleeding and iatrogenic retinal breaks, early and delayed VHs, recurrent retinal detachment, neovascular glaucoma, and anterior hyaloid fibrovascular proliferation. ${ }^{4,13}$

In the last 10 years, $\mathrm{SGV}$ became the common practice for TRD. ${ }^{14,15}$ However, doubt has been raised whether diabetic TRD, in which complex intraocular manipulations are needed and complications occur more commonly, can be equally managed by SGV. ${ }^{1,13,15}$

SGV has several advantages in treatment of diabetic TRD as well as other diseases. The prominent ones are smaller incisions without peritomy, self-sealing sclerotomies that heal faster, diminished conjunctival scarring that allows repeat surgeries, decreased postoperative inflammation, and a decrease in induced astigmatism that may facilitate visual recovery. ${ }^{9,11,13,16}$ Moreover, because the cutting tip is closer to the edge of the device, the vitrector facilitates segmentation, dissection, and removal of the fibrovascular membranes in PDR. ${ }^{9}, 13$ The smaller lumen diameter also improves fluidics, allowing for better fluid stability and less tissue movement or incarceration. Better fluidics increase the safety of intraoperative maneuvers. ${ }^{17,18}$

The purpose of this study is to retrospectively evaluate the outcomes and complications of TRD in patients with PDR managed by PPV. Moreover, we tried to find out whether patients with poor visual acuity (VA) had different VA gain than patients with VA better than 20/200.

\section{Patients and methods Subjects}

This retrospective study included a consecutive series of patients who underwent PPV with $23 \mathrm{G}$ and $25 \mathrm{G}$ instrumentation for the management of TRD due to PDR between October 2011 and November 2013 at a single center by three senior surgeons.

Indications for vitrectomy were TRD involving or threatening the macula or unresolved VH combined with TRD.
Patients with previous vitrectomy were excluded from the study. Approval to review the patients' data was obtained from the local ethics committee.

\section{Methods}

The medical files were reviewed of all patients who underwent PPV for the management of TRD due to PDR. The following data were retrieved: patient's age, sex, medical background, past hospitalizations and blood tests, preoperative and postoperative Snellen VA, intraocular pressure (IOP) and results of slit-lamp examination of the anterior segment and fundus, surgical reports, and intraoperative and postoperative complications.

Snellen VAs were converted into the logarithm of the minimum angle of resolution (LogMAR) score for data analysis.

Thirteen $(54.2 \%)$ of the cases were conducted under regional anesthesia and eleven $(45.8 \%)$ under general anesthesia. All surgeries were performed with the Stellaris Vitreoretinal Surgical System (Bausch \& Lomb Incorporated, Bridgewater, NJ, USA) and a wide-angle viewing system. The surgeon could select either $25 \mathrm{G}$ or $23 \mathrm{G}$ instrument depending on the patient's preference. Diabetic membrane removal was achieved either with a cutter or with the curved microscissors. In all cases, the vitreous was removed up to the far periphery, involving meticulous removal around the ports and the infusion line. At the completion of the surgery, the peripheral retina was examined using scleral depression. Drainage retinotomy was performed in cases of subretinal fluid. In cases where a retinal tear was noted, fluid air exchange was performed. Endolaser panretinal photocoagulation up to the far periphery and laser around holes and tears were performed in all patients. At the end of surgery, air, gas, or silicone oil tamponade was used. Sclerotomies were checked for leakage, and if needed, a vicryl suture was applied.

All surgeries were performed by experienced vitreoretinal surgeons. Those patients undergoing combined cataract vitrectomy surgery had their crystalline lens removed using phacoemulsification techniques. A clear corneal approach was used in all cases, and a posterior chamber intraocular lens was inserted to the bag when possible.

\section{Statistical analysis}

Analyses were performed using SPSS for Windows (SPSS, Inc., Chicago, IL, USA). Data were expressed as mean \pm standard deviation. Wilcoxon signed-rank test was used to compare preoperative and postoperative LogMAR VA. 
Furthermore, Pearson's correlation coefficient was used to search for an existing relationship between the preoperative and postoperative VA, and Fisher's exact test was used to search for a preoperative VA under which the probability to improve VA would be lower. A $P$-value $<0.05$ was used to mark statistical significance.

\section{Results}

Twenty-four eyes of 21 PDR patients (58.3\% males) who had undergone PPV for the management of TRD during the aforementioned period were included in this study. Patients' characteristics are summarized in Table 1.

Of the 24 surgeries performed, 23 utilized the $23 \mathrm{G}$ instrumentation and one utilized the $25 \mathrm{G}$ instrumentation. Fourteen surgeries $(58.3 \%)$ were performed on the left eye. Fifteen eyes $(62.5 \%)$ had $\mathrm{VH}$ at the time of surgery. Eleven cases $(45.8 \%)$ underwent combined cataract and vitrectomy surgery.

The average hemoglobin $\mathrm{A}_{1 \mathrm{C}}$ value as measured in patients' routine blood tests since 2010 was $11.5 \%$ (6.4\%$17.7 \%$, normal values $4.0 \%-5.7 \%$ ). Each patient's maximal hemoglobin $\mathrm{A}_{1 \mathrm{C}}$ was taken for average calculation purposes as a marker of the unbalanced diabetes. Seventeen patients were diagnosed with chronic hypertension (81.0\%) and 16 were diagnosed with hyperlipidemia $(76.2 \%)$. The mean number of emergency hospitalizations for nonophthalmologic reasons during the follow-up period was $2.3(0-7)$.

Preoprerative examination revealed detached macula in 12 of the cases $(50 \%)$ and tractions threatening the macula in nine additional cases $(37.5 \%)$. The remaining three cases $(12.5 \%)$ involved a thick, long-standing, nonclearing $\mathrm{VH}$

Table I Characteristics of 2 I patients who underwent small-gauge vitrectomy during $20 \mathrm{I} \mathrm{I}-20 \mathrm{I} 3$

\begin{tabular}{ll}
\hline Number of eyes & 24 \\
Number of patients & $2 \mathrm{I}$ \\
Mean follow-up (months), mean \pm SD & $13.3 \pm 7.6(4-30)$ \\
(range) & \\
Sex (male/female) & $13 / 8$ \\
Mean age (years), mean \pm SD (range) & $55.9 \pm 12.5(22-79)$ \\
Diabetes mellitus (type I/type 2) & $3 / 18$ \\
Mean maximal HbA ${ }_{\text {IC }} \%$ & $11.5 \% \pm 2.7 \%$ \\
(mean \pm SD), range, \% ${ }^{\mathrm{a}}$ & $(6.4 \%-17.7 \%)$ \\
Hypertension, $\mathrm{n}(\%)$ & $17(8 \mathrm{I} .0)$ \\
Hyperlipidemia, $\mathrm{n}(\%)$ & $16(76.2)$ \\
Chronic renal failure, $\mathrm{n}(\%)$ & $10(47.6)$ \\
Ischemic heart disease, $\mathrm{n}(\%)$ & $9(42.9)$ \\
Mean number of emergency & $2.3 \pm 2.1(0-7)$ \\
hospitalizations, $20 \mathrm{I} \mathrm{I}-20 \mathrm{I} 3$ (range) & \\
\hline
\end{tabular}

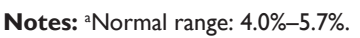

Abbreviations: $\mathrm{HbA}_{I C}$, hemoglobin $\mathrm{A}_{\mathrm{IC}} ; \mathrm{SD}$, standard deviation. with tractional detachment in the posterior pole that could be demonstrated only by an ultrasound examination. Six of the cases $(25 \%)$ were diagnosed with diabetic macular edema prior to the surgery.

Intraoperative and postoperative complications are described in Table 2. Retinal breaks were observed in ten of the cases $(41.7 \%)$ and seven of them reported as iatrogenic $(29.2 \%)$. Retinal and vitreal hemorrhages occurred in nine of the operations $(37.5 \%)$.

Intraocular tamponade was perfluoropropane $(\mathrm{C} 3 \mathrm{~F} 8)$ in eight cases $(33.3 \%)$, sulfur hexafluoride (SF6) in five cases $(20.8 \%)$, silicone oil in three cases $(12.5 \%)$, and air in one case $(4.2 \%)$.

A statistically significant improvement in mean LogMAR VA values was found between preoperative and final follow-up examination $(1.48 \pm 0.65$ and $1.05 \pm 0.65$, respectively, $P=0.04$; Figure 1). Moreover, $62.5 \%$ of the cases had a final VA $\geq 20 / 200$, and $75 \%$ showed an improvement of $\geq 0.3$ LogMAR with 95\% confidence interval of 53\%-90\% exact by nominal confidence interval. Only $12.5 \%$ of the cases had decreased VA at the end of the follow-up period when compared with the preoperative VA. One case regressed to no light perception due to uncontrolled glaucoma, which was known prior to the surgery. A correlation between preoperative and postoperative VA was not found $(r=-0.1$, $P=0.7$; Figure 2).

On an attempt to find a cutoff point for the feasibility of the operation, the cases were divided into two subgroups. Those with preoperative VA of LogMAR $\leq 1$ (ten patients) and those with LogMAR $>1$ (14 patients). Sixty percent of those in the first group showed improvement in their

Table 2 Intraoperative and postoperative complications in 24 cases that underwent small-gauge vitrectomy during 20I I-2013

\begin{tabular}{ll}
\hline & Percentage \\
\hline Intraoperative complications & \\
Retinal breaks & 41.7 \\
Vitreous hemorrhage & 37.5 \\
20 G conversion & 8.3 \\
Unplanned cataract extraction & 8.3 \\
Choroidal detachment & 4.2 \\
Postoperative complications & \\
Vitreous hemorrhage & 54.2 \\
Glaucoma & 20.8 \\
Reoperation & 20.8 \\
Retinal detachment & 12.5 \\
Dislocated PCIOL & 4.2 \\
Epiretinal membrane & 29.2 \\
Macular hole & 8.3 \\
\hline
\end{tabular}

Abbreviation: $\mathrm{PCIOL}$, posterior chamber intraocular lens. 


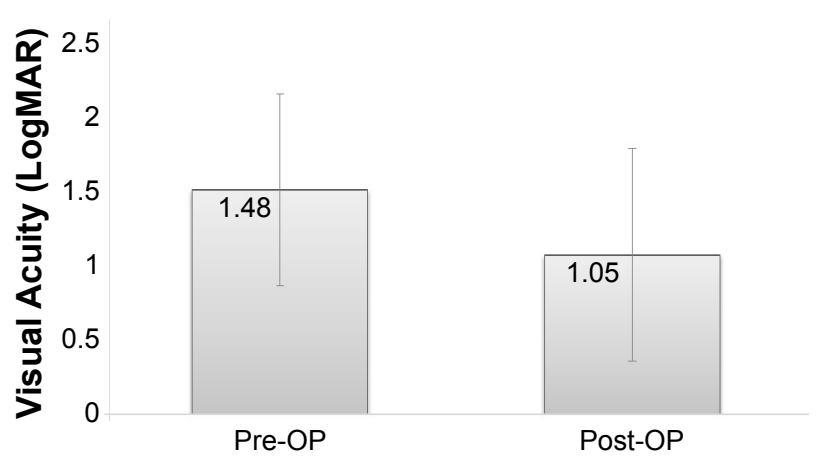

Figure I Postoperative versus preoperative visual acuity (LogMAR). Note: $P<0.05$, significant difference compared with preoperative visual acuity. Abbreviations: LogMAR, logarithm of the minimum angle of resolution; OP, operative.

final postoperative VA comparing with $86 \%$ in the second group. Fisher's exact test did not show a significant difference between the groups; however, there is a trend toward a greater probability to improve the postoperative VA with a decreased preoperative VA $(P=0.19)$.

Postoperative complications (Table 2 ) of various degrees were documented in 21 cases $(87.5 \%)$. Although VH occurred in 13 cases (54.2\%), an additional surgery to clear the vitreous was performed only in one case, in which a very thick hemorrhage developed several days after the first surgery. In the other cases, the hemorrhage was absorbed spontaneously or with the aid of antivascular endothelial growth factor (anti-VEGF) injection. A second operation was performed in five cases (20.8\%), of which three were due to nonabsorbing subretinal fluids, one due to the aforementioned $\mathrm{VH}$, and one owing to a dislocated intraocular lens. Newly diagnosed postoperative glaucoma occurred in five cases (20.8\%). None of the cases had new neovascularization of the iris or the angle. Additional postoperative treatment with anti-VEGF intraocular injections due to VH or macular

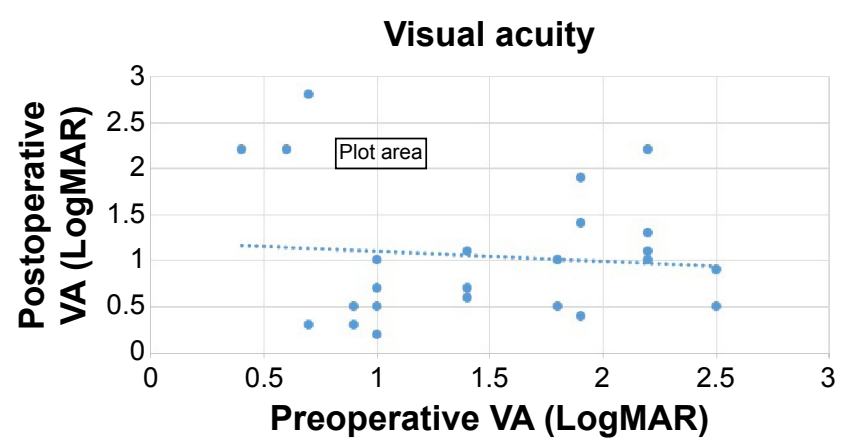

Figure $\mathbf{2}$ Linear regression analysis of the relationship between preoperative and postoperative visual acuity (VA).

Note: No correlation was observed between these two variants (Pearson's correlation coefficient, $r=-0.1, P=0.7$ ).

Abbreviation: LogMAR, logarithm of the minimum angle of resolution. edema was given in six of the cases (25.0\%). There were no cases of postoperative hypotony or endophthalmithis.

\section{Discussion}

Although SGV became the mainstay of the management of diabetic TRD in the last decade, questions still remain regarding its ability to overcome the complexity of this procedure. ${ }^{10,13}$ The current study is focusing on the subgroup of diabetic TRD patients, trying to accentuate the advantages of surgery along with the accompanying complications and risks.

The results of the present study showed that VA significantly improved at the end of the follow-up period and thus suggested that $\mathrm{SGV}$ is indeed an effective treatment for this subgroup.

A total of $62.5 \%$ of the cases had a final VA $\geq 20 / 200$ and $75 \%$ showed an improvement of $\geq 0.3 \operatorname{LogMAR}$. Only $12.5 \%$ of cases experienced a deterioration in VA. These results are supported by other studies. ${ }^{13,15,19}$

Moreover, high rates $(87.5 \%)$ of primary retinal reattachment were found in the present study. The ultimate reattachment rate after repeated surgeries, as evaluated in the final examination, was $100 \%$. These findings are in accordance with previous studies. . $^{2,3,13}$

There was no statistically significant correlation between the preoperative and the postoperative VA. However, a trend was found toward greater probability to have a postoperative VA improvement with a preoperative VA of $\operatorname{LogMAR}>1$ than with $\operatorname{LogMAR} \leq 1$. This might be a subject for further research in larger future studies trying to elaborate whether there are cases in which surgery should be recommended.

On the other hand, a considerable amount of intraoperative and postoperative complications occurred in this study. Retinal breaks were found in higher rates (41.7\%) compared with other studies. Kumar et a $1{ }^{15}$ reported an incidence of $16 \%$ when using the $23 \mathrm{G}$ and $20 \%$ when using the $25 \mathrm{G}$. Similar rates of $21 \%$ were noted by Oshima et al. ${ }^{13}$ Such differences may be attributed to the higher percentage of patients with preoperative VH found in the present study (62.5\%), which may have prevented the diagnosis of some existing retinal breaks. Kumer et al ${ }^{15}$ reported an incidence of $24 \%$ in the $23 \mathrm{G}$ group and $20 \%$ in the $25 \mathrm{G}$ group, whereas Oshina et al ${ }^{13}$ reported an incidence of $45 \%$.

In one prospective study on retinal breaks in $\mathrm{SGV}$, a rate of $41.1 \%$ of retinal breaks was reported in diabetic patients with traction retinal detachment. ${ }^{20}$ Latrogenic retinal breaks were previously reported to be related to the complexity of the case with rates of up to $78 \%$ in complex fibrovascular proliferation. ${ }^{21}$ 
When comparing rates of retinal breaks in PDR in $20 \mathrm{G}$ versus $23 \mathrm{G}$ vitrectomy, lower rates of retinal breaks were reported in the latter. ${ }^{13,22,23}$

Postoperative VH was a major complication in this study (54.3\%). However, only one single case justified a second surgical intervention due to its thickness and occurrence several days postoperatively. In all other cases, postoperative VHs were reabsorbed spontaneously or with the assistance of intraocular anti-VEGF injection. Ozone et $\mathrm{al}^{10}$ reported a $22 \%$ incidence of postoperative $\mathrm{VH}$ in their study following $25 \mathrm{G}$ vitrectomies in diabetic patients with $\mathrm{VH}$, TRD, or fibrovascular proliferation. A similar rate of $21.4 \%$ was noted by Altan et al. ${ }^{19}$ The higher incidence found in the present study may be attributed to the patients' poorly controlled underlying diabetes. Additionally, our study included all vitreous bleeding regardless of volume or thickness, while other studies may have disregarded negligible bleedings.

Another important postoperative complication in our study was elevated IOP in five cases $(20.8 \%)$, which was unrelated to neovascularization of the iris or the anterior chamber angle. Both Wu et $\mathrm{al}^{24}$ and Chang ${ }^{25}$ described similar findings in their retrospective studies of vitrectomized eyes; however, they excluded cases with severe PDR. Other studies reported lower incidence of this complication..$^{26,27}$ The mechanism suggested for this complication was increased oxidative stress in the anterior chamber after vitrectomy that damaged the trabecular meshwork. ${ }^{24,25}$ Elevated oxidative stress is considered an important factor in the development of diabetic retinopathy ${ }^{28}$ and may therefore also amplify the risk of developing high IOP after vitrectomy by the aforementioned mechanism.

In the present study, no case of endophthalmitis was documented. Increased experience with SGV, as demonstrated by more complete vitrectomy, careful checking for wound leakage, closure of the sclerotomies by massage, and the use of angled sclerotomies dramatically decreased the incidence of endophthalmitis. ${ }^{29}$

Hypotony is another common immediate postoperative complication following SGV, with incidence of up to $16 \%{ }^{30,31}$ However, none of the patients included in this study developed hypotony postoperatively. This could be attributed to the common use of intraocular tamponade, the use of biplanar oblique sclerotomies, and the liberal use of sutures whenever suspected leakage or incomplete closure occurred at the end of the surgery.

In most cases, inadequate treatment of diabetes over the years may have been the cause of severe retinopathy and may explain the high number of associated cardiovascular diseases and hospitalizations, and low compliance of patients to treatment.

\section{Conclusion}

In conclusion, the current study demonstrates that patients with diabetic TRD benefit from surgical intervention. Moreover, SGV is an effective and safe procedure for the management of this complex disease owing to the experience and improved instruments and techniques gained over the years. The complications associated with SGV seem to be less pronounced than thought previously.

The limitations of the current study include its small size, its retrospective nature, and lack of control group. A prospective randomized controlled trial would be beneficial and strengthen the results shown in the current study.

\section{Disclosure}

The authors report no conflicts of interest in this work.

\section{References}

1. Gupta B, Sivaprasad S, Wong R, et al. Visual and anatomical outcomes following vitrectomy for complications of diabetic retinopathy: the DRIVE UK study. Eye. 2012;26(4):510-516.

2. Tao Y, Jiang YR, Li XX, Gao L, Jonas JB. Long-term results of vitrectomy without endotamponade in proliferative diabetic retinopathy with tractional retinal detachment. Retina. 2010;30(3):447-451.

3. Qamar RM, Saleem MI, Saleem MF. The outcomes of pars plana vitrectomy without endotamponade for tractional retinal detachment secondary to proliferative diabetic retinopathy. Int J Ophthalmol. 2013;6(5): 671-674.

4. Newman DK. Surgical management of the late complications of proliferative diabetic retinopathy. Eye. 2010;24(3):441-449.

5. O'Malley C, Heintz RM Sr. Vitrectomy with an alternative instrument system. Ann Ophthalmol. 1975;7(4):585-588, 591-594.

6. Fujii GY, De Juan E Jr, Humayun MS, et al. A new 25-gauge instrument system for transconjunctival sutureless vitrectomy surgery. Ophthalmology. 2002;109(10):1807-1812. [discussion 1813].

7. Lesnoni G, Rossi T, Gelso A. 25 Gauge vitrectomy instrumentation: a different approach. Semin Ophthalmol. 2004;19(1-2):49-54.

8. Eckardt C. Transconjunctival sutureless 23-gauge vitrectomy. Retina. 2005;25(2):208-211.

9. Arumi JG, Boixadera A, Martinez-Castillo V, Corcostegui B. Transconjunctival sutureless 23-gauge vitrectomy for diabetic retinopathy. Review. Curr Diabetes Rev. 2009;5(1):63-66.

10. Ozone D, Hirano Y, Ueda J, Yasukawa T, Yoshida M, Ogura Y. Outcomes and complications of 25-gauge transconjunctival sutureless vitrectomy for proliferative diabetic retinopathy. Ophthalmologica. 2011;226(2): 76-80.

11. Fine HF, Iranmanesh R, Iturralde D, Spaide RF. Outcomes of 77 consecutive cases of 23-gauge transconjunctival vitrectomy surgery for posterior segment disease. Ophthalmology. 2007;114(6):1197-1200.

12. Bourla DH, Bor E, Axer-Siegel R, Mimouni K, Weinberger D. Outcomes and complications of rhegmatogenous retinal detachment repair with selective sutureless 25-gauge pars plana vitrectomy. Am J Ophthalmol. 2010; 149(4):630-634.e1.

13. Oshima Y, Shima C, Wakabayashi T, et al. Microincision vitrectomy surgery and intravitreal bevacizumab as a surgical adjunct to treat diabetic traction retinal detachment. Ophthalmology. 2009;116(5):927-938. 
14. Farouk MM, Naito T, Sayed KM, et al. Outcomes of 25-gauge vitrectomy for proliferative diabetic retinopathy. Graefes Arch Clin Exp Ophthalmol. 2011;249(3):369-376.

15. Kumar A, Duraipandi K, Gogia V, Sehra SV, Gupta S, Midha N. Comparative evaluation of 23- and 25-gauge microincision vitrectomy surgery in management of diabetic macular traction retinal detachment. Eur J Ophthalmol. 2014;24(1):107-113.

16. Thompson JT. Advantages and limitations of small gauge vitrectomy. Surv Ophthalmol. 2011;56(2):162-172.

17. Steel DH, Charles S. Vitrectomy fluidics. Ophthalmologica. 2011; 226(suppl 1):27-35.

18. Dugel PU, Zhou J, Abulon DJ, Buboltz DC. Tissue attraction associated with 20-gauge, 23-gauge, and enhanced 25-gauge dual-pneumatic vitrectomy probes. Retina. 2012;32(9):1761-1766.

19. Altan T, Acar N, Kapran Z, Unver YB, Ozdogan S. Transconjunctival 25-gauge sutureless vitrectomy and silicone oil injection in diabetic tractional retinal detachment. Retina. 2008;28(9):1201-1206.

20. Ehrlich R, Goh YW, Ahmad N, Polkinghorne P. Retinal breaks in smallgauge pars plana vitrectomy. Am J Ophthalmol. 2012;153(5):868-872.

21. Carter JB, Michels RG, Glaser BM, De Bustros S. Iatrogenic retinal breaks complicating pars plana vitrectomy. Ophthalmology. 1990;97(7):848-853. [discussion 854].

22. Issa SA, Connor A, Habib M, Steel DH. Comparison of retinal breaks observed during 23 gauge transconjunctival vitrectomy versus conventional 20 gauge surgery for proliferative diabetic retinopathy. Clin Ophthalmol. 2011;5:109-114.
23. Park DH, Shin JP, Kim SY. Comparison of clinical outcomes between 23-gauge and 20-gauge vitrectomy in patients with proliferative diabetic retinopathy. Retina. 2010;30(10):1662-1670.

24. Wu L, Berrocal MH, Rodriguez FJ, et al. Intraocular pressure elevation after uncomplicated pars plana vitrectomy: results of the Pan American Collaborative Retina Study Group. Retina. 2014;34(10):1985-1989.

25. Chang S. LXII Edward Jackson lecture: open angle glaucoma after vitrectomy. Am J Ophthalmol. 2006;141(6):1033-1043.

26. Koreen L, Yoshida N, Escariao P, et al. Incidence of, risk factors for, and combined mechanism of late-onset open-angle glaucoma after vitrectomy. Retina. 2012;32(1):160-167.

27. Toyokawa N, Kimura H, Matsumura M, Kuroda S. Incidence of late-onset ocular hypertension following uncomplicated pars plana vitrectomy in pseudophakic eyes. Am J Ophthalmol. 2015;159(4):727-732.

28. Behl T, Kaur I, Kotwani A. Implication of oxidative stress in progression of diabetic retinopathy. Surv Ophthalmol. Epub 2015.

29. Scott IU, Flynn HW Jr, Acar N, et al. Incidence of endophthalmitis after 20 -gauge vs 23 -gauge vs 25 -gauge pars plana vitrectomy. Graefes Arch Clin Exp Ophthalmol. 2011;249(3):377-380.

30. Hsu J, Chen E, Gupta O, Fineman MS, Garg SJ, Regillo CD. Hypotony after 25-gauge vitrectomy using oblique versus direct cannula insertions in fluid-filled eyes. Retina. 2008;28(7):937-940.

31. Kellner L, Wimpissinger B, Stolba U, Brannath W, Binder S. 25-Gauge vs 20-gauge system for pars plana vitrectomy: a prospective randomised clinical trial. Br J Ophthalmol. 2007;91(7):945-948.
Clinical Ophthalmology

\section{Publish your work in this journal}

Clinical Ophthalmology is an international, peer-reviewed journal covering all subspecialties within ophthalmology. Key topics include: Optometry; Visual science; Pharmacology and drug therapy in eye diseases; Basic Sciences; Primary and Secondary eye care; Patient Safety and Quality of Care Improvements. This journal is indexed on Submit your manuscript here: http://www.dovepress.com/clinical-ophthalmology-journal

\section{Dovepress}

PubMed Central and CAS, and is the official journal of The Society of Clinical Ophthalmology (SCO). The manuscript management system is completely online and includes a very quick and fair peer-review system, which is all easy to use. Visit http://www.dovepress.com/ testimonials.php to read real quotes from published authors. 